\title{
Uso de métodos anticoncepcionais entre adolescentes universitários
}

\author{
Use of contraceptive methods among university adolescents
}

El uso de metodos anticonceptivos en adolescentes universitarios

\author{
Aline Salheb Alves', Maria Helena Baena de Moraes Lopes ${ }^{1}$ \\ 'Universidade Estadual de Campinas, Faculdade de Ciências Médicas, Departamento de Enfermagem. Campinas, SP
}

Submissão: 13/02/2007

Aprovação: 02/08/2007

\section{RESUMO}

Objetivou-se identificar o uso de métodos anticoncepcionais entre adolescentes, ingressantes de uma universidade pública paulista e descrever o perfil desses universitários Quanto a algumas características sócio-demográficas. Foi utilizado um Questionário e a amostra foi composta por 295 adolescentes. Relataram ter iniciado atividade sexual 48,8\%. A idade média para o início foi aos 17 anos, e a maioria relatou ter usado um método contraceptivo. O método mais utilizado foi o preservativo e este em combinação com a pílula, principalmente. Observou-se Que os adolescentes buscam informações, seja através de profissionais da saúde, professores, ou através de livros, com destaque para a mídia. Conclui-se que os universitários iniciam atividade sexual mais tardiamente e buscam conhecimento sobre anticoncepção.

Descritores: Adolescente; Sexualidade; Anticoncepção.

\section{ABSTRACT}

The objective of this study was to identify the use of contraceptive methods among teenagers at a public University in São Paulo, as well as to describe their profile concerning social and demographic characteristics. A Questionnaire was used and the sample was composed by 295 teenagers. The initiation of sexual activity was reported by $48,8 \%$. The mean age was seventeen and most of them reported that they used some type of contraceptive method. The method used with highest frequency was the preservative, and this in combination with the pill. It could be observed that teenagers seek information through health professionals, teachers or books, and mainly media. It was concluded that the students had sexual initiation late and seek information about contraception.

Descriptors: Adolescent; Sexuality; Contraception.

\section{RESUMEN}

El objetivo de este trabajo es identificar el uso de los métodos anticonceptivos entre adolescentes, alumnos de una universidad paulista, y describir su perfil basados en algunas características socio-demográficas. Se utilizó un cuestionario aplicado a 295 adolescentes. El 48,8\% relataron haber iniciado actividad sexual. En media, la edad para el inicio son los 17 años y la mayoría afirma haber usado métodos anticonceptivos. El más utilizado fue, principalmente, el preservativo combinado con la píldora. Se observó que los adolescentes buscan información consultando a profesionales de la salud, profesores o a través de libros, y sobretodo por los medios de comunicación. Se concluye que los universitarios inician su actividad sexual más tarde y buscan conocimiento sobre métodos anticonceptivos.

Descriptores: Adolescente; Sexualidad; Anticoncepción. 


\section{INTRODUÇÃO}

A sexualidade, presente em toda a trajetória de vida do ser humano, busca sua afirmação na adolescência. Este é um fenômeno Que aparece na maioria das culturas ocidentais, uma vez Que o mercado de trabalho exige uma especialização cada vez maior e, conseqüentemente, um maior período de preparação dos jovens, impedindo com isso que eles ingressem no mercado de trabalho e assumam a tão desejada autonomia ${ }^{(1)}$.

No entanto, o desenvolvimento da sexualidade nem sempre é acompanhado de um amadurecimento afetivo e cognitivo, o Que torna a adolescência uma etapa de extrema vulnerabilidade a riscos, os Quais estão muito ligados às características próprias do desenvolvimento psico-emocional dessa fase da vida ${ }^{(2)}$.

As vivências da sexualidade expõem os adolescentes à gravidez precoce, ao aborto, à AIDS e a outras doenças sexualmente transmissíveis (DST), Que podem comprometer o projeto de vida ou até mesmo a própria vida ${ }^{(2)}$. Estudos mostram que, cada vez mais, o início da atividade sexual dá-se na adolescência, devido a exposição às influências urbanas e às mudanças dos valores tradicionais $^{(3-5)}$.

No Brasil, a prevalência de uso dos métodos anticoncepcionais é alta, porém concentrada na esterilização tubária (laQueadura) e na pílula anticoncepcional, utilizadas por $40 \%$ e $21 \%$ das mulheres, respectivamente ${ }^{(6)}$. Entre os adolescentes, os métodos mais utilizados são o preservativo masculino e a pílula anticon-cepcional ${ }^{(3,7)}$. Porém, apesar dos diversos estudos realizados com adolescentes ${ }^{(4,5,8)}$, poucos pesQuisaram a parcela universitária ${ }^{(7.9)}$.

Optou-se por desenvolver um estudo com a população adolescente devido à necessidade de maior conhecimento deste grupo etário em relação ao seu processo de decisão, às influências recebidas, bem como o contexto e a perspectiva deste público, para o desenvolvimento de estratégias de intervenções específicas. Além disso, com universitários, já Que apresentam um diferencial em relação ao perfil educacional.

Para tanto foi desenvolvida a pesquisa intitulada "Locus de controle e conhecimento, atitude e prática para o uso de pílula e preservativo entre adolescentes universitários". No presente artigo, objetivou-se identificar o uso de métodos anticoncepcionais (MAC) entre adolescentes, alunos ingressantes de uma universidade pública do Estado de São Paulo e descrever seu perfil Quanto a algumas características sócio-demográficas.

\section{MÉTODO}

Tratou-se de um estudo descritivo e transversal, Que utilizou abordagem Quantitativa.

A população do estudo foi composta por adolescentes universitários, de ambos os sexos, ingressantes de uma universidade pública do Estado de São Paulo. A universidade em Questão oferece vagas para 58 cursos de graduação. Soma-se um total de 2830 vagas para os alunos ingressantes, sendo Que 1940 são para o período diurno. A média de alunos por sala de aula é 50,5 , segundo informações da diretoria acadêmica da universidade ${ }^{(10)}$.

Uma vez Que a Organização Mundial da Saúde (OMS) define como adolescentes as pessoas com idade entre dez e 19 anos, definição adotada no Brasil pelo Programa de Saúde do Adolescente do Ministério da Saúde ${ }^{(\text {II) }}$, foram incluídos na estudo os alunos com idade igual ou inferior a 19 anos. Como é esperado que estudantes do período noturno tenham perfil diferente daquele do período diurno, porque alguns optam por esse horário por serem trabalhadores, para a pesquisa foram excluídos os cursos do período noturno (20 cursos) e de outros campi (07 cursos). Também não foram abordados os alunos de cursos em Que não foi obtida autorização do(a) coordenador(a) de graduação para a coleta de dados.

Foi utilizado cálculo de tamanho amostral para coeficiente de correlação ${ }^{(12)}$. Com base no percentual por sexo dos alunos ingressantes no ano de 2006 (60\% masculino e 40\% feminino) e nas variáveis de interesse, considerou-se para o cálculo do tamanho amostral o coeficiente de correlação (r) de 0,20, alfa 0,05 (bilateral) e beta 0,10 (poder de 90\%), obtendo-se o tamanho amostral de 259 alunos.

Foi encaminhada uma carta a todos os coordenadores de graduação dos cursos da Universidade, solicitando autorização para a coleta de dados. Um e-mail também foi enviado, contendo as mesmas informações da carta e cópia digital dos instrumentos de coleta de dados. Após um mês, o mesmo e-mail foi reenviado aos coordenadores Que não enviaram resposta. Ao final, foi obtida autorização de 19 cursos: Artes Cênicas, Ciências Biológicas, Educação Física, Enfermagem, Farmácia, Fonoaudiologia, Ciências da Terra, Geografia, Geologia, Engenharia Agrícola, Engenharia Civil, Engenharia de Alimentos, Engenharia de Computação, Engenharia Elétrica, Engenharia Mecânica, Engenharia Química, Ciências Econômicas, História e Pedagogia.

Foi utilizado um Questionário para obter dados referentes a características sócio-demográficas (idade, sexo, cor da pele, religião, atividade remunerada, renda familiar, presença de companheiro, com Quem mora), características da vida sexual (idade de início da atividade sexual, uso de MAC na primeira relação sexual, idade de início de uso de MAC, uso atual de MAC e número de gravidezes). O Questionário foi submetido à avaliação de três juízes, pesquisadoras da área de saúde da mulher, as Quais fizeram sugestões relevantes. Além disso, o Questionário foi pré-testado com um grupo de 13 adolescentes, estudantes de outro campus da mesma universidade.

Para garantir o sigilo dos respondentes, cada curso foi identificado através de um código alfabético (por exemplo: $A A, A B, B A, B B$ e assim por diante) e fez-se uma listagem contendo os códigos e os respectivos cursos. Além disso, os Questionários foram identificados apenas por números para viabilizar seu processamento.

Os adolescentes foram abordados em suas respectivas salas de aula, após autorização do professor, e responderam o Questionário sob supervisão da pesquisadora, no período compreendido entre março e junho de 2006. Foram distribuídos o Questionário e o termo de consentimento livre e esclarecido para aqueles Que manifestaram desejo em participar do estudo. O tempo médio para responder o Questionário foi de 15 minutos.

Foram cumpridos os termos da Resolução n 196 do Conselho Nacional de Saúde (1996). A pesquisa foi submetida ao Comitê de Ética em Pesquisa da Faculdade de Ciências Médicas - FCM UNICAMP e aprovada (Parecer Projeto no 5 16/2005).

Foi criado um banco de dados no programa Epi Info versão 3.3.2 e os dados foram inseridos e depois conferidos antes de se proceder 
a análise. Calcularam-se as freqüências absoluta (n) e percentual (\%) das variáveis categóricas. As variáveis contínuas foram analisadas descritivamente. Foi usado o teste de Wilcoxon para comparar as idades de início das atividades sexuais e de uso de MAC. Foi estabelecido o nível de significância de 5\%.

\section{RESULTADOS}

Fizeram parte da casuística 295 adolescentes universitários. No ano de 2006, a idade predominante dos ingressantes nesta universidade foi 18 anos (32,5\%), sendo a maioria do sexo masculino $(58,3 \%)$, de cor branca $(76,7 \%)$, sem trabalho remunerado $(82,6 \%)$ e com renda familiar superior a 10 salários mínimos (56,3\%).

Observa-se no Quadro I as características sócio-demográficas do grupo estudado. A idade variou de 17 a 19 anos, mas a maioria dos adolescentes $(82,7 \%)$ esteve concentrada na faixa etária dos 18 aos 19 anos, era do sexo masculino $(51,9 \%)$, consideraram-se brancos $(79,7 \%)$, denominaram-se católicos $(50,2 \%)$, não trabalhavam $(92,2 \%)$, viviam com a família ou amigos $(82,1 \%)$ e tinham renda familiar de 6 a 10 salários-mínimos ou mais (59,3\%). Comparando-se esses dados com os de todos os ingressantes em 2006, nota-se Que as proporções se mantêm, com exceção da renda familiar.

Observa-se no Quadro 2 que 66,8\% da amostra não possuíam companheiro, e pouco mais da metade ( 15 I ou $51,2 \%$ ) relatou não ter iniciado atividade sexual. Separando-se por gênero, a diferença foi pequena, $50 \%$ do sexo masculino e $53,6 \%$ do feminino não haviam iniciado atividade sexual. Referiram ter iniciado 144 $(48,8 \%)$, sendo $52,8 \%$ do sexo masculino e $43,7 \%$ do feminino. Houve 5 não respondentes dessa Questão.

Quanto ao uso de MAC na primeira relação sexual, 91,7\% afirmaram Que utilizaram e a idade média de início do uso do MAC foi de 16,8 $( \pm 1,38)$, mínima de 13 e máxima de 19. Porém uma proporção expressiva $(29,2 \%)$ não respondeu a idade Que iniciou a

Quadro 1. Características sócio-demográficas de adolescentes ingressantes de uma universidade pública do Estado de São Paulo ( $n=295)$ - março-junho de 2006.

\begin{tabular}{|c|c|c|c|}
\hline \multirow{2}{*}{ Característica } & \multirow{2}{*}{ Categoria } & \multicolumn{2}{|c|}{ Freeüência } \\
\hline & & $\mathrm{n}$ & $\%$ \\
\hline \multirow[t]{3}{*}{ Idade } & 17 & 51 & 17,3 \\
\hline & 18 & 135 & 45,8 \\
\hline & 19 & 109 & 36,9 \\
\hline \multirow[t]{2}{*}{ Sexo } & Masculino & 153 & 51,9 \\
\hline & Feminino & 142 & 48,1 \\
\hline \multirow[t]{6}{*}{ Cor } & Branca & 235 & 79,7 \\
\hline & Parda & 28 & 9,5 \\
\hline & Amarela & 23 & 7,8 \\
\hline & Preta & 6 & 2,0 \\
\hline & Indígena & 1 & 0,3 \\
\hline & Outra & 2 & 0,7 \\
\hline \multirow[t]{7}{*}{ Religião } & Católica & 148 & 50,2 \\
\hline & Espírita & 30 & 10,2 \\
\hline & Evangélica & 22 & 7,5 \\
\hline & Judaica & 1 & 0,3 \\
\hline & Não tem & 74 & 25,0 \\
\hline & Outra & 18 & 6,1 \\
\hline & Não responderam & 2 & 0,7 \\
\hline \multirow[t]{2}{*}{ Trabalho Remunerado } & Não & 272 & 92,2 \\
\hline & Sim & 23 & 7,8 \\
\hline \multirow{5}{*}{$\begin{array}{l}\text { Renda Familiar (em salários } \\
\text { mínimos) }\end{array}$} & 0 & 12 & 4,1 \\
\hline & $1-5$ & 56 & 19,0 \\
\hline & $6-10$ & 110 & 37,3 \\
\hline & $>10$ & 65 & 22,0 \\
\hline & Não responderam & 52 & 17,6 \\
\hline \multirow[t]{6}{*}{ Moradia } & Família & 146 & 49,6 \\
\hline & Amigos & 96 & 32,5 \\
\hline & Sozinho & 39 & 13,2 \\
\hline & Companheiro & 1 & 0,3 \\
\hline & Outros & 10 & 3,4 \\
\hline & Não responderam & 3 & 1,0 \\
\hline
\end{tabular}


Quadro 2. Características sexuais de adolescentes ingressantes de uma universidade pública do Estado de São Paulo - março-junho de 2006.

\begin{tabular}{|c|c|c|c|}
\hline \multirow[b]{2}{*}{ Característica } & \multirow[b]{2}{*}{ Categoria } & \multicolumn{2}{|c|}{ Freqüência } \\
\hline & & $\mathrm{n}$ & $\%$ \\
\hline \multirow{3}{*}{$\begin{array}{l}\text { Presença de Companheiro } \\
(n=295)\end{array}$} & Não & 197 & 66,8 \\
\hline & Sim & 91 & 30,8 \\
\hline & Não responderam & 07 & 2,4 \\
\hline \multirow{3}{*}{$\begin{array}{l}\text { Uso de MAC na Primeira } \\
\text { Relação Sexual }(n=144)\end{array}$} & Sim & 132 & 91,7 \\
\hline & Não & 07 & 4,9 \\
\hline & Não responderam & 05 & 3,4 \\
\hline \multirow{4}{*}{$\begin{array}{l}\text { Freqüência das Relações } \\
\text { Sexuais }(n=144)\end{array}$} & De vez em Quando & 56 & 38,9 \\
\hline & FreQüentemente & 35 & 24,3 \\
\hline & No momento não têm & 33 & 22,9 \\
\hline & Não responderam & 20 & 13,9 \\
\hline \multirow{5}{*}{$\begin{array}{l}\text { Número de Relações } \\
\text { Sexuais/Semana }(n=35)\end{array}$} & $01 \mathrm{vez}$ & 10 & 28,6 \\
\hline & 02 vezes & 09 & 25,7 \\
\hline & 03 vezes & 07 & 20,0 \\
\hline & 04 vezes & 05 & 14,3 \\
\hline & 05 vezes ou mais & 04 & 11,4 \\
\hline \multirow{3}{*}{$\begin{array}{l}\text { Uso de MAC atualmente } \\
(n=144)\end{array}$} & Sim & 118 & 81,9 \\
\hline & Não & 15 & 10,5 \\
\hline & Não responderam & 11 & 7,6 \\
\hline
\end{tabular}

Quadro 3. Métodos anticoncepcionais utilizados e motivos para o não uso de MAC entre adolescentes ingressantes de uma universidade pública do Estado de São Paulo - março-junho de 2006.

\begin{tabular}{|c|c|c|c|}
\hline \multirow[b]{2}{*}{ Característica } & \multirow[b]{2}{*}{ Categoria } & \multicolumn{2}{|c|}{ FreQüência } \\
\hline & & $\mathrm{n}$ & $\%$ \\
\hline \multirow{13}{*}{$\begin{array}{l}\text { MAC Utilizado na } \\
\text { Primeira Relação } \\
\text { Sexual }(n=144)\end{array}$} & Preservativo Masculino & 95 & 66,0 \\
\hline & Preserv. Masc. + Pílula & 21 & 14,5 \\
\hline & Pílula & 05 & 3,4 \\
\hline & Preserv. Masc. + Tabelinha & 02 & 1,4 \\
\hline & Preservativo Feminino & 02 & 1,4 \\
\hline & Pílula do Dia Seguinte & 02 & 1,4 \\
\hline & Pílula + Preserv. Masc. + Coito Interrompido & 01 & 0,7 \\
\hline & Pílula + Preserv. Masc. + Pílula do Dia Seguinte + Tabela & 01 & 0,7 \\
\hline & Preserv. Masc. + Píl. do Dia Seguinte & 01 & 0,7 \\
\hline & Coito Interrompido & 01 & 0,7 \\
\hline & Outro & 01 & 0,7 \\
\hline & Nenhum & 07 & 4,9 \\
\hline & Não responderam & 05 & 3,5 \\
\hline \multirow{3}{*}{$\begin{array}{l}\text { Motivo Para o Não } \\
\text { Uso na Primeira } \\
\text { Relação Sexual }(\mathrm{n}=7)\end{array}$} & Não pensou na hora & 05 & 71,4 \\
\hline & Não gosta & 01 & 14,3 \\
\hline & Vergonha & 01 & 14,3 \\
\hline \multirow[t]{11}{*}{ MAC Atual $(\mathrm{n}=144)$} & Preservativo Masculino & 55 & 38,2 \\
\hline & Preserv. Masc. + Pílula & 27 & 18,7 \\
\hline & Pílula & 25 & 17,4 \\
\hline & Preserv. Masc. + Pílula + Tabelinha & 03 & 2,1 \\
\hline & Preserv. Masc. + Tabelinha & 02 & 1,4 \\
\hline & Pílula + Coito Interrompido & 02 & 1,4 \\
\hline & Preservativo Feminino & 01 & 0,7 \\
\hline & Preserv. Fem. + Preserv. Masc. & 01 & 0,7 \\
\hline & Tabelinha & 01 & 0,7 \\
\hline & Nenhum & 15 & 10,4 \\
\hline & Não responderam & 12 & 8,3 \\
\hline \multirow{5}{*}{$\begin{array}{l}\text { Motivo Para o Não } \\
\text { Uso Atualmente } \\
(n=15)\end{array}$} & Não tem relação sexual & 06 & 40,0 \\
\hline & As relações sexuais são esporádicas & 03 & 20,0 \\
\hline & O(a) companheiro(a) usa MAC & 02 & 13,3 \\
\hline & É responsabilidade do(a) parceiro(a) & 01 & 6,7 \\
\hline & Não responderam & 03 & 20,0 \\
\hline
\end{tabular}


Quadro 4. Influência para a escolha do método anticoncepcional entre adolescentes ingressantes de uma universidade pública do Estado de São Paulo ( $\mathrm{n}=129)$ - março-junho de 2006.

\begin{tabular}{|lcc|}
\hline & & Freeüência \\
Categoria * & $\mathbf{n}$ & \% \\
\hline Informação de livros, revistas, TV e Internet & 39 & 30,2 \\
Informação dada por médico/ profissional da saúde & 39 & 30,2 \\
Indicação de médico/ profissional da saúde & 30 & 23,2 \\
Sugestão do companheiro (a) & 28 & 21,7 \\
Sugestão de familiar & 25 & 19,4 \\
Informação dada por professor & 16 & 12,4 \\
Sugestão de amigo (a) & 12 & 9,3 \\
Outro & 07 & 5,4 \\
Não receberam influência & 15 & 11,6 \\
Não responderam & 15 & 11,6 \\
\hline
\end{tabular}

* Não soma 100\% porque mais de uma alternativa pôde ser escolhida.

Quadro 5. Distribuição dos adolescentes universitários de acordo com a idade de início da atividade sexual e idade de uso de MAC ( $n=295$ ) - março-junho de 2006.

\begin{tabular}{|lcccc|}
\hline \multirow{2}{*}{ Idades } & \multicolumn{2}{c|}{ Início Atividade Sexual } & \multicolumn{2}{c|}{ Início Uso do MAC } \\
\cline { 2 - 5 } & $\mathbf{n}$ & $\mathbf{\%}$ & $\mathbf{n}$ & \% \\
\hline Não iniciaram & 151 & 51,2 & - & - \\
12 & 03 & 1,0 & 00 & 00 \\
13 & 03 & 1,0 & 02 & 1,4 \\
14 & 03 & 1,0 & 04 & 2,8 \\
15 & 21 & 7,1 & 11 & 7,6 \\
16 & 27 & 9,2 & 20 & 13,9 \\
17 & 45 & 15,3 & 36 & 25,0 \\
18 & 23 & 7,8 & 17 & 11,8 \\
19 & 14 & 4,7 & 12 & 8,3 \\
Não responderam & 05 & 1,7 & 42 & 29,2 \\
\hline
\end{tabular}

utilização de MAC.

As relações sexuais eram esporádicas (38,9\%) e $81,9 \%$ usavam MAC naquele momento. Não foi referido nenhum caso de gravidez nem de aborto no grupo estudado.

No Quadro 3 são apresentados os MAC utilizados na primeira relação sexual, e vemos o uso predominante do preservativo masculino (66\%), freeüentemente combinado com outros métodos, principalmente com a pílula. A pílula, como único método, foi pouco utilizada $(3,4 \%)$.

Como método atual, em uso no momento da pesquisa, o preservativo foi o mais citado $(38,2 \%)$, com freqüência utilizado juntamente com outros métodos, porém seu uso é menor em comparação com a época da primeira relação. $\mathrm{O}$ uso isolado da pílula $(17,4 \%)$ como método anticoncepcional aumentou em relação ao início da vida sexual.

Dentre os motivos mais referidos para o não uso de MAC, o mais citado na primeira relação sexual foi "não pensar na hora". Quanto ao não uso no momento da coleta de dados, os motivos mais referidos foram não ter relação sexual no momento e a esporadicidade das relações sexuais.

No Quadro 4 é apresentado o Que influenciou os 129 adolescentes Que usavam MAC a optarem pelo método atual. Nota-se a importância do médico ou profissional de saúde na informação ou indicação de MAC (53,4\%), bem como dos meios de comunicação (30,2\%).
O Quadro 5 mostra a distribuição dos adolescentes universitários de acordo com a idade de início da atividade sexual e idade de uso de MAC. Comparando-se estas idades através do teste de Wilcoxon para amostras relacionadas, verificou-se diferença média entre as idades de 0,37 anos, com desvio padrão de $\pm 1,11$, diferença mínima de zero e máxima de sete anos. O p-valor calculado foi de 0,07 , ou seja, não houve diferença significativa entre as idades, apenas uma tendência. Cinco $(3,5 \%)$ adolescentes iniciaram o uso do MAC antes de iniciar atividade sexual e $12(8,3 \%)$ começaram a utilizar MAC após o a primeira relação sexual.

\section{DISCUSSÃO}

No presente estudo, vê-se que os adolescentes buscaram informação para a escolha do método contraceptivo, mas também receberam influência do companheiro, familiar ou de amigos.

Cerca de um terço buscou informação por meio de livros, revistas, televisão ou internet. As tecnologias de informação e comunicação na internet expandiram os limites do ensino e, além disso, a leitura eletrônica ocupa a preferência entre os adolescentes ${ }^{(13)}$, fato Que nos direciona euanto às estratégias mais adequadas a serem utilizadas com esta população. Chama a atenção o fato de Que apenas $12,4 \%$ foram influenciados por professores na sua decisão, o Que sugere a falta ou inadeQuação da educação sexual nas escolas. 
De fato, a educação sexual concentrada na transmissão de informação científica não tem resultado em comportamento saudável e, dessa forma, outras fontes de informação devem ser testadas ${ }^{(14)}$.

A mesma parcela $(30,2 \%)$ recebeu informação de médico ou profissional da saúde para a escolha de um MAC, o Que demonstra preocupação e interesse com o assunto. Entretanto, nem sempre o profissional auxilia no processo de decisão, informando sobre todos os métodos; freqüentemente ele decide pelo cliente. A exemplo disso, um estudo realizado com 563 estudantes da Universidade Federal de Pernambuco (UFPE) sobre comportamento de risco para a AIDS, evidenciou Que nas consultas são prescritos contraceptivos orais sem nenhuma orientação Quanto ao uso do preservativo(15).

No estudo atual, apenas $24,3 \%$ referiu ter recebido influência de familiares para a escolha do método. Ainda hoje, há barreiras para o diálogo entre pais e filhos no Que diz respeito às Questões de sexualidade. O desconforto Que muitos adultos sentem Quando discutem tais assuntos pode advir do sentimento de Que a sexualidade é algo impuro ou sem importância. Pesquisas americanas recentes mostraram Que um terço dos adolescentes relataram Que não receberam instruções formais sobre métodos contraceptivos antes dos 18 anos de idade. Apenas metade das mulheres entre 18-19 anos e um terço dos homens disseram Que conversaram com seus pais sobre tais Questões antes dos $18 \operatorname{anos}^{(16)}$.

No presente estudo, 51 ,2\% ainda não tinham iniciado atividade sexual, e a idade média na primeira relação foi por volta dos 17 anos, tanto para o grupo masculino Quanto para o feminino. Outro estudo $^{(7)}$ confirma Que estudantes universitários iniciam mais tardiamente sua vida sexual. Este fenômeno recentemente observado pode ser uma evidência das transformações ocorridas no comportamento sexual da população brasileira, por conta da entrada da mulher no mercado de trabalho, da crescente escolarização, do uso generalizado de MAC, e do contexto da AIDS, Que tornou as Questões da sexualidade mais proeminentes $^{(17)}$. Além disso, esse início tardio da atividade sexual também pode ser explicado pelo projeto de vida diferenciado daqueles adolescentes Que têm por meta o estudo universitário.

Provavelmente o objetivo do ingresso em uma universidade pública, a priorização dos estudos, a maior preocupação com o futuro e a aQuisição de responsabilidade do universitário tenham favorecido tal situação. Reforça esta idéia o fato de Que não foi relatado nenhum caso de aborto ou de gravidez, embora não se possa descartar a possibilidade de omissão desta informação por parte dos Que não responderam ou Que não Quiseram participar da pesquisa.

Num estudo realizado com 563 universitários da UFPE, a idade média de início da atividade sexual para o sexo masculino foi 15,7 e para o sexo feminino, 18,4 $4^{(15)}$. No estudo realizado entre universitários de uma universidade pública paulista ${ }^{(7)}$, as idades foram 17 anos no grupo formado pelos homens e 18 anos no das mulheres. Diferentemente, em estudo realizado com 356 adolescentes Que freqüentavam um ambulatório de Saúde do Adolescente da Universidade Estadual do Rio de Janeiro, a idade da primeira relação sexual se concentrou na faixa dos $14-15$ anos ${ }^{(18)}$. Este início precoce talvez seja explicado pela diferença de perfil destes adolescentes daQueles Que são universitários: Quase metade deles era portadora de DSTs e apresentava atraso escolar maior Que dois anos. Vê-se aí a importância da escolaridade e de um objetivo Que norteie a vida dos adolescentes, para postergar o início da atividade sexual até um momento mais adequado.

As idades de início da atividade sexual e de início de uso de MAC foram aos 17 anos, entretanto não diferiram significativamente. Porém um número expressivo $(29,2 \%)$ não respondeu a idade em Que iniciaram o uso de MAC, talvez por não se lembrarem, o Que provavelmente indica Que o uso não foi concomitante com o início das atividades sexuais. Apesar de ser peQueno o número de adolescentes Que referiram não utilizar MAC na primeira relação sexual, este fato não deixa de ser preocupante dada à gravidade do fato, principalmente considerando-se que $10,5 \%$ dos adolescentes responderam Que não estavam utilizando MAC no momento da coleta de dados.

No Brasil, a prevalência de uso dos métodos anticoncepcionais é alta, porém concentrada na esterilização tubária (laQueadura) e na pílula anticoncepcional, utilizadas por $40 \%$ e $21 \%$ das mulheres, respectivamente $^{(6)}$. Já entre os adolescentes, os métodos mais utilizados são o preservativo masculino e a pílula anticoncepcional $^{(5,7)}$, o Que foi confirmado no estudo atual.

Em correspondência com pesQuisas envolvendo adolescentes ${ }^{(3,7)}$, no presente estudo, o MAC mais freqüentemente utilizado foi o preservativo masculino, referido por $84 \%$ no primeiro intercurso sexual e por 61 , 1\% como método atual. Porém foi utilizado não apenas como método único, mas também em combinação com outros, principalmente com a pílula. Esse fato leva-nos a considerar Que houve a preocupação tanto com a gravidez indesejada Quanto com as DSTs. Reforça essa idéia o fato de Que duas adolescentes utilizaram 2 ou 3 métodos na primeira relação sexual além do preservativo. $\mathrm{O}$ uso da pílula do dia seguinte como método único ou associado a outros, também evidencia a preocupação em se evitar a gravidez. Por outro lado, embora pouco freqüente, observase também o uso de MAC de baixa eficácia e/ou Que requerem conhecimento amplo da fisiologia, sendo utilizado por alguns adolescentes, como é o caso da tabelinha e do coito interrompido.

Estudo realizado com 952 estudantes de graduação de uma universidade pública estadual de São Paulo, com idade entre 17 e 24 anos, revelou Que um número elevado de entrevistados referiu fazer uso de contraceptivos. Os principais métodos usados eram o preservativo e a pílula, usados separadamente ou combinados. Constatou-se também Que a proporção de usuários de pílula tendia a aumentar entre os entrevistados Que referiram o namoro, indicando uma tendência de o preservativo ser gradativamente substituído pela pílula. Outro importante achado deste mesmo estudo diz respeito ao aborto. Evidenciou-se que a maioria das gestações entre os universitários foi finalizada por um aborto provocado ${ }^{(7)}$. No presente estudo não houve relato de gravidez e/ou aborto, mas de forma semelhante ao estudo citado, observou-se que no início da vida sexual, um maior número de adolescentes usavam o preservativo, mas Que no momento da coleta de dados, embora o uso de preservativo fosse o mais freeüente, houve aumento do uso de pílula.

O preservativo ainda é pouco utilizado e seu uso é infreQüente, havendo uma enorme lacuna entre o nível de conhecimento e o uso efetivo. Além disso, foram encontrados baixos índices de uso de preservativo na faixa etária de 15 a 19 anos entre adolescentes do Rio de Janeiro que freqüentavam um ambulatório de Saúde do Adolescente $^{(18)}$. Em contraste com esse dado, um inquérito 
nacional $^{(19)}$ realizado com 6000 indivíduos de 15 a 54 anos evidenciou Que os mais jovens ( 15 a 24 anos) foram os que mais usaram o preservativo.

Estudos têm constatado um alto nível de conhecimento dos métodos anticoncepcionais pelos adolescentes e jovens ${ }^{(7,19)}$, de modo Que o não-uso não deve ser relacionado diretamente com a falta de informação. Entre os fatores Que influenciam o não-uso de MAC estão, principalmente, a esporadicidade e a falta de planejamento das relações sexuais ${ }^{(7)}$. O estudo atual confirma isso Quando $71,4 \%$ dos adolescentes justificaram o não uso de MAC na primeira relação sexual por não terem pensado na hora, o Que demonstra a imaturidade psíquica desta faixa etária. A esporadicidade das relações sexuais também foi citada. Chama a atenção, a atitude manifesta por alguns adolescentes de atribuir a responsabilidade do uso do MAC ao parceiro, Quando para maior segurança sexual e reprodutiva, ambos deveriam utilizá-los, uma vez Que, a exemplo dos métodos mais usados, o preservativo masculino tem média eficácia e a pílula não protege contra as DSTs.

O preservativo, eneuanto protetor, contribui para reduzir o risco de infecções adeuiridas através da exposição à região cervical, vaginal, vulvar e anal, ressaltando-se Que esta eficácia está diretamente associada ao uso correto e regular, bem como à Qualidade do preservativo. Seu uso é, ainda hoje, permeado por tabus e preconceitos. Crenças na promoção da promiscuidade ou na redução da sensibilidade, não aceitação pelo parceiro, idéias equivocadas sobre a eficácia, embaraço relacionado à compra do preservativo e não tê-lo disponível no momento, fortalecem a relutância de muitos para o uso correto e sistemático ${ }^{(20)}$.

Detectou-se Que os adolescentes estavam interessados na busca de informações Quantos aos métodos, seja através de profissionais da área da saúde, professores, ou através de livros, com destaque para a mídia. O desenvolvimento de estratégias mais voltadas para esta população, como por exemplo, o maior uso da internet, pode ser um recurso valioso.

A educação é uma função inerente aos profissionais de saúde, e embora aparentemente simples e fácil, é um processo complexo. É necessário desenvolver o senso de responsabilidade individual e grupal para Que sejam possíveis mudanças de comportamento, baseando-se em aceitação e não em obrigação(21). É importante a educação sexual sistematizada, Que desmistifica as crenças negativas, e associa o uso dos métodos anticoncepcionais ao prazer resultante da segurança Que eles proporcionam. As pessoas precisam ser sensibilizadas Quanto aos riscos reais Que elas correm, para Que ocorram mudanças de comportamentos e atitudes, para um exercício sexual seguro.

\section{CONCLUSÕES}

Frente ao exposto, verificou-se Que, neste grupo de adolescentes universitários, o método mais utilizado foi o preservativo usado separadamente ou em combinação com a pílula e/ou outros MAC. Muitos ainda não tinham iniciado a atividade sexual e o início da atividade sexual foi tardio Quando comparado com adolescentes não universitários, de acordo com a literatura.

Não houve diferença significativa entre a idade de início da atividade sexual e a de início de uso de MAC, entretanto houve uma pequena parcela de adolescentes que correu os riscos inerentes ao sexo desprotegido. Observou-se Que estes adolescentes buscam informações por meio de diversas fontes, mas que a influência de professores é pequena.

\section{REFERÊNCIAS}

I. Farias MA. Adolescência - do Que estamos falando? [citado em: 17 jul 2005]. Disponível em: URL: http:// www.brazilpednews.org.br/setem99/ar9903.htm

2. Saito MI. Adolescência, cultura, vulnerabilidade e risco. Pediatria 2000; 22(3): 217-9.

3. Martins LBM. Conhecimento, atitude e prática sobre métodos anticoncepcionais, prevenção de DST/AIDS em adolescentes de escolas públicas e privadas do município de São Paulo [dissertação]. Campinas (SP): Faculdade de Ciências Médicas - UNICAMP; 2005.

4. Belo MAVB, Silva JLP. Conhecimento, atitude e prática sobre métodos anticoncepcionais entre adolescentes gestantes. Rev Saúde Pública 2004; 38(4): 479-87.

5. Schor N, Lopez FA. Adolescência e anticoncepção. Estudo de conhecimento e uso em puérperas internadas por parto ou aborto. Rev Saúde Pública 1990; 26(6): 506-I 1 .

6. Duarte GA. Contracepção e aborto: perspectiva masculina [dissertação]. São Paulo (SP): Faculdade de Saúde Pública, USP; 2000.

7. Pirotta KC, Schor N. Intenções reprodutivas e práticas de regulação da fecundidade entre universitários. Rev Saúde Pública 2004; 38(4): 495-502.

8. Martins LBM, Costa-Paiva L, Osis MID, Sousa MH, Neto AMP, Tadini V. Conhecimento sobre métodos anticoncepcionais

por estudantes adolescentes. Rev. Saúde Pública 2006; 40(1): 57-64.

9. Gadelha LM, Lopes CM. A influência do hábito sexual e o risco de infecção pelo HIV: conhecimento de universitários recém-ingressos na UFAC, ano 1999. DST - I Bras Doenças Sex Transm 2000; 12(2): 19-30.

10. Comvest. Comissão permanente para vestibulares 2006. [citado em: 24 jun 2004]. Disponível em: URL: http:// www.comvest.unicamp.br/estatisticas/perfil/perfil2006.pdf

11. World Health Organization. Child and adolescent health and development [on line]. [cited 2001 sep 28]. Avaiable from: URL: http://www.who.int/child-adolescent-health/OVERVIEW/AHD/

12. Hulley SB, Cummings SR. Designing Clinical Research. Baltimore (PA): Williams \& Wilkins; 1988.

13. Moro ELS, Souto GP, Estabel LB. A influência da internet nos hábitos de leitura do adolescente. 2005. [citado em: I I out 2006]. Disponível em: URL: http://www.eci.ufmg.br/gebe/ downloads/3 13.pdf

14. Borichovitch E. Fatores associados à não utilização de anticoncepcionais na adolescência. Rev Saúde Pública 1992; 26 (6): 437-43.

15. Carneiro RM, Ludermir AB, Duarte MSM, Moreira MFA, Campelo DEC, Melo LMP. Comportamento de risco para Aids entre estudantes universitários. A experiência da UFPE. 
Uso de métodos anticoncepcionais entre adolescentes universitários

An Fac Med Univ Fed Pernamb 1999; 44(2): 1 1 3-7.

16. Nelson AL, Shields WL. Healthy sexuality. Contraception 2005; 71:399-401.

17. Borges ALV, Schor N. Início da vida sexual na adolescência e relações de gênero, um estudo transversal em São Paulo. Brasil, 2002. Cad Saúde Pública 2005; 2 I (2): 499-507.

18. Taquette SR, Vilhena MM, Paula MC. Doenças sexualmente transmissíveis na adolescência: estudo de fatores de risco. Rev Soc Bras Med Trop 2004; 34(3): 21 0-4.

19. Szwarcwald CL, Júnior $A B$, Pascom AR, Júnior PRS. PesQuisa de conhecimento, atitudes e práticas na população brasileira de 15 a 54 anos, 2004. Bol Epidemiol AIDS e DST 2004; I(I): 18-24.

20. Gir E, Duarte G, Carvalho MJ. Opinião de universitários sobre o uso do condom e sua influência no exercício da sexualidade. Med Ribeirão Preto 1997; 30: 100-5.

21. Gir E, Moriya TM, Hayashida M, Duarte G, Machado AA. Medidas preventivas contra a Aids e outras doenças sexualmente transmissíveis conhecidas por universitários da área da saúde. Rev Latino-am Enfermagem 1999; 7(1): I 1-7. 